\title{
Erratum
}

\section{Correction of Figure: Fertility preservation for adolescent and young adult cancer patients in Japan}

Yodo Sugishita, Nao Suzuki

In the published article, "Yodo Sugishita, Nao Suzuki. Fertility preservation for adolescent and young adult cancer patients in Japan. Obstet Gynecol Sci 2018;61(4):443-452. https://doi.org/10.5468/ogs.2018.61.4.443," Fig. 2 was published incorrectly.

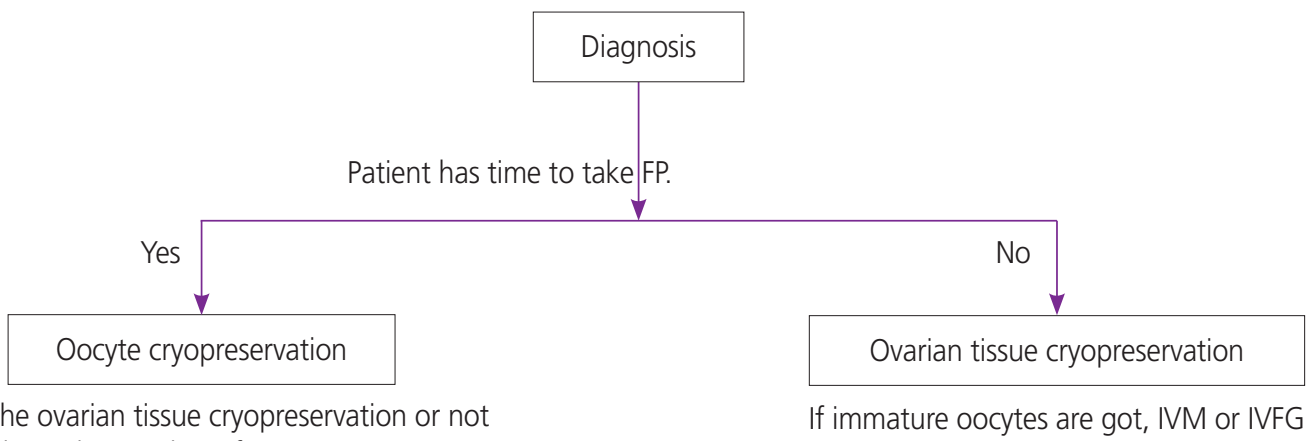

Adding the ovarian tissue cryopreservation or not It depends on the number of oocytes

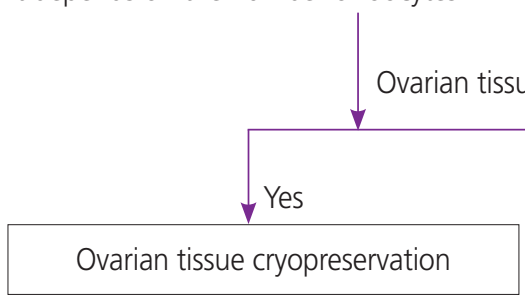

If immature oocytes are got, IVM or IVFG

Fig. 2. Male fertility preservation on young cancer patients in adolescent and young adult generation. FP, fertility preservation; IVM, in vitro maturation; IVFG, in vitro follicle growth; ASAP, as soon as possible.

Fig. 2 should be corrected as follows. 


\section{Obstetrics \& Gynecology Science}

Yodo Sugishita, et al. Erratum: How to consider fertility preservation in AYA

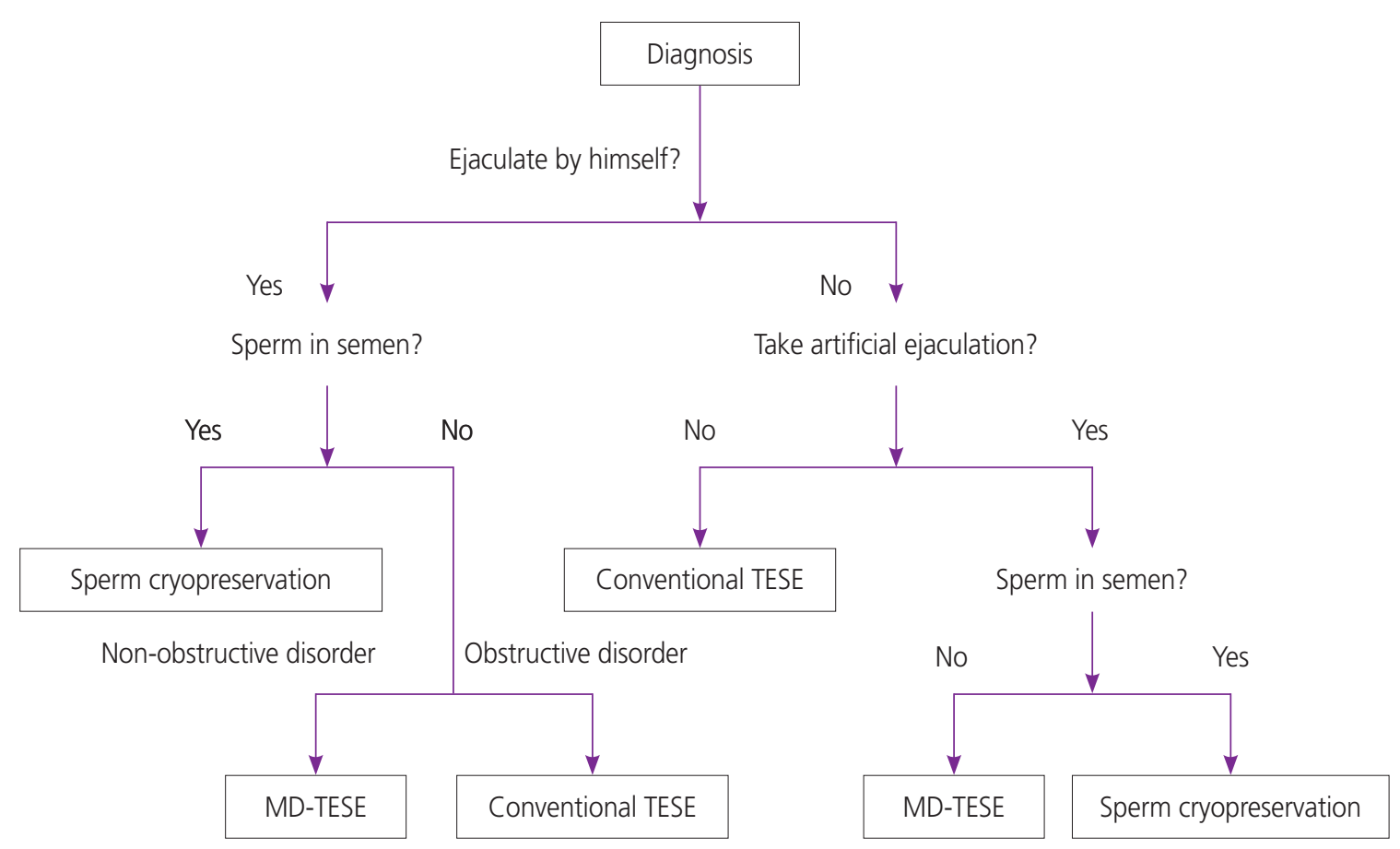

Fig. 2. Male fertility preservation on young cancer patients in adolescent and young adult generation. TESE, testicular sperm extraction; MD-TESE, microdissection-testicular sperm extraction. 